\title{
Sistemas de información geográficas aplicados en salud líneas de investigación
}

\author{
Gustavo D. Buzai, Claudia A. Baxendale, Nicolás Caloni, \\ María del Rosario Cruz, Hugo Delfino, Gimena Mora y Noelia \\ Principi
}

\section{Resumen:}

La estandarización completa de las metodologías geográficas cuantitativas en el ambiente computacional finalizó al comenzar el siglo XXI. El uso de Sistemas de Información Geográfica (SIG) y Sistemas de Ayuda a la Decisión Espacial (SADE) presentan desarrollos que sintetizan procedimientos centrales del análisis espacial. En este contexto la Geografía de la Salud ha sido una de las líneas de investigación con mayores posibilidades de aplicación en el estudio de distribuciones espaciales de características poblacionales, enfermedades y la oferta de servicios orientados a la atención de la población.

Se presenta un panorama global y actualizado de la relación entre SIG y Geografía de la Salud como marco para la presentación de diferentes ejemplos de resultados obtenidos de una década de trabajo. Los resultados obtenidos apoyan el proceso de toma de decisiones en investigación, planificación y gestión en aspectos espaciales de la salud.

Palabras clave: Geografía de la Salud / Geografía Médica / Geografía de los Servicios Sanitarios / Análisis Espacial / Geografía Aplicada.

\section{Abstract:}

The complete standardization of geographical quantitative methodologies in computing environment ended at the beginning of the XXI century. The using of Geographic Information Systems (GIS) and Spatial Decision Support Systems (SDSS) present developments that synthesize core spatial analysis procedures.

In this context Geography of Health has been one of the research lines with the greatest potential for applications in the study of spatial distributions of population 
characteristics, disease and offer services geared to the attention of the population. Wepresentacomprehensiveandupdatedoverview oftherelationshipbetween Geographical Information Systems and Geography of Health as a framework for the presentation of different examples of results of a decade of work. The results support the decision making process in research, planning and management in spatial aspects of health.

Keywords: Health Geography, / Medical Geography / Geography of Health Services / Spatial Analysis / Applied Geography

Gustavo D. Buzai (Director); Claudia A. Baxendale; Nicolás Caloni; María del Rosario Cruz: Hugo Delfino;, Gimena Mora y Noelia Principi. Universidad Nacional de Luján, Grupo de Estudios sobre Geografía y Análisis Espacial con Sistemas de Información Geográfica (GESIG). Argentina. (www.gesig-proeg.com.ar), (gesig-proeg@unlu.edu.ar). 


\section{INTRODUCCIÓN}

Hace tres décadas hemos comenzado a recorrer un camino que concluyó con la estandarización completa de las metodologías geográficas cuantitativas en el ambiente computacional.

La Geografía como ciencia generó metodologías y técnicas que brindaron sustento al desarrollo de las tecnologías geoinformáticas y ellas son utilizadas ampliamente para logar la resolución de problemas socioespaciales (Buzai y Baxendale, 2011 y 2012). De estas tecnologías sobresalen los Sistemas de Información Geográfica (SIG) y los Sistemas de Ayuda a la Decisión Espacial (SADE).

En este contexto, de acuerdo al trabajo de Gatrell (2003), la Geografía de la Salud ha sido una de las líneas temáticas que ha podido tener mayor receptividad en el uso de tecnologías geodigitales basadas en la cuantificación. Ha incorporado un amplio espectro de metodologías para el estudio de las distribuciones y asociaciones espaciales entre enfermedades y condiciones sociales (Geografía Médica), y de las localizaciones e interacciones entre la población y los centros de atención (Geografía de los Servicios).

La presente ponencia tiene como objetivo presentar, en estos dos grandes bloques temáticos, la línea de investigación en Geografía de la Salud desarrollada en el Grupo de Estudios sobre Geografía y Análisis Espacial con Sistemas de Información Geográfica (GESIG) de la Universidad Nacional de Luján.

\section{EL ALCANCE TEMÁTICO}

La temática se vincula de forma general al estudio de la salud formado por cuatro dimensiones (Howe, 1985): (1) biología humana, (2) ambiente, (3) forma de vida, y (4) sistema de asistencia médica.

De acuerdo con las tendencias actuales la Geografía como ciencia ligada al uso de las nuevas tecnologías geoinformáticas puede brindar utilidad principal en dos de estas dimensiones.

La dimensión 2 definiría el campo de la Geografía Médica la cual pone acento en la definición clásica de la relación hombre-medio, principalmente se ana- 
lizan aquellas cuestiones del entorno que afectan la salud de la población.

La dimensión 4 definiría el campo de la Geografía de los Servicios de Salud considerando los centros de atención médica como puntos de oferta distribuidos entre la población de demanda.

Ambas dimensiones, ligadas a las líneas de abordaje temático, son las que comprenden el campo de la Geografía de la Salud (Olivera, 1994). Por lo tanto es de esperarse que ambas líneas tengan una importante autonomía en sus modelos de problemas y, al mismo tiempo, en los métodos aplicados para encontrar soluciones.

\section{APLICACIONES EN GEOGRAFIA MÉDICA}

La aplicación de SIG+SADE en estudios de Geografía Médica son amplios y variados. Para el estudio de distribuciones puntuales se incluye el análisis de tendencia central o el cálculo de densidades kernel con la consiguiente capacidad de superposición temática. Para el estudio de distribuciones areales se incluye el análisis multivariado por cartografía temática, el análisis bivariado y trivariado dentro del análisis exploratorio de datos espaciales (ESDA), búsqueda de correspondencia por superposición cartográfica y técnicas de evaluación multicriterio. Procedimientos de clasificación multivariada en la búsqueda de áreas homogéneas, áreas de concentración espacial, análisis de autocorrelación espacial global y local, y la aplicación de métodos de regresión múltiple y simple ajustada geográficamente.

Los procedimientos aplicados en los proyectos de GESIG (Buzai, 2007a, 2007b, 2009 y 2013; Cruz, 2012) son los siguientes:

Aplicación del Análisis Exploratorio de Datos Espaciales (ESDA) en el estudio de la distribución espacial univariada de enfermedades. 
Figura 1: Luján. Box-map de enfermedades infecciosas

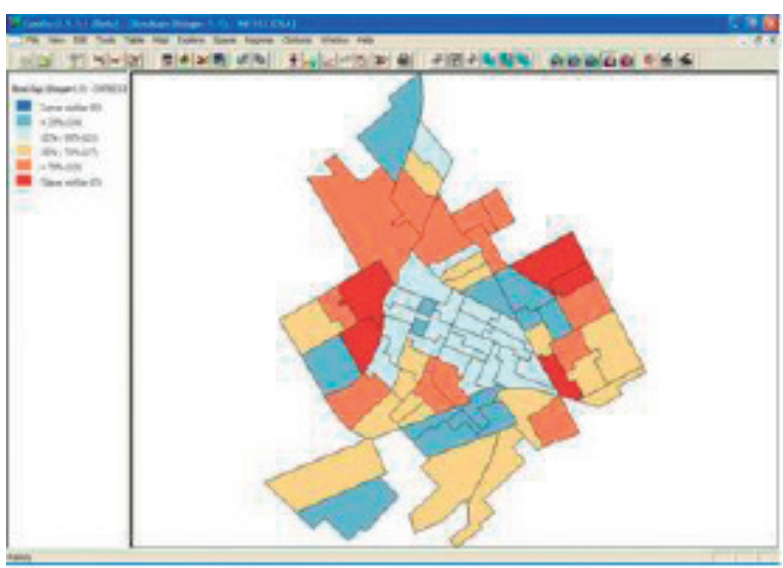

Aplicación del Análisis Exploratorio de Datos Espaciales (ESDA) en el estudio de la distribución espacial bivariada de enfermedades con características sociales.

Figura 2. Luján. Distribución espacial de la selección del cuadrante ++

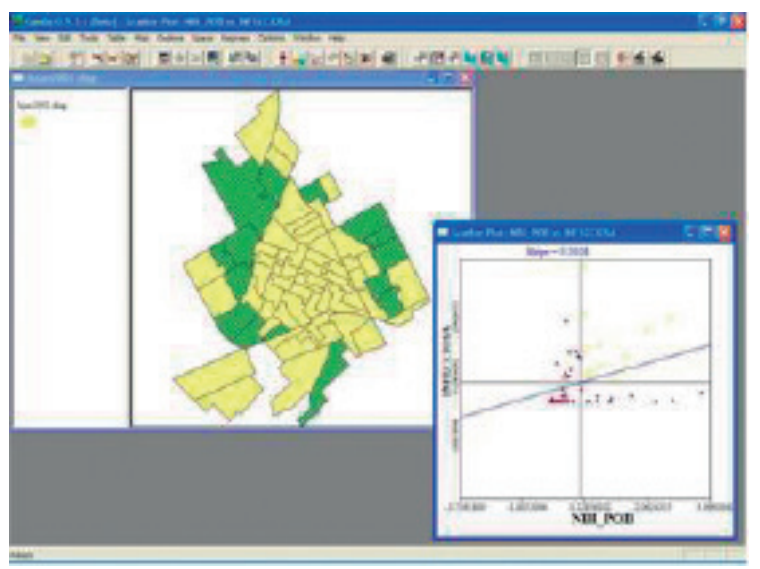

Aplicación del Análisis Exploratorio de Datos Espaciales (ESDA) en el estudio de la distribución espacial trivariada de enfermedades. 
Figura 3. Luján: Configuración espacial de la selección enfermedades: Infecciosas (x) - Respiratorias (y) - Digestivas (z)

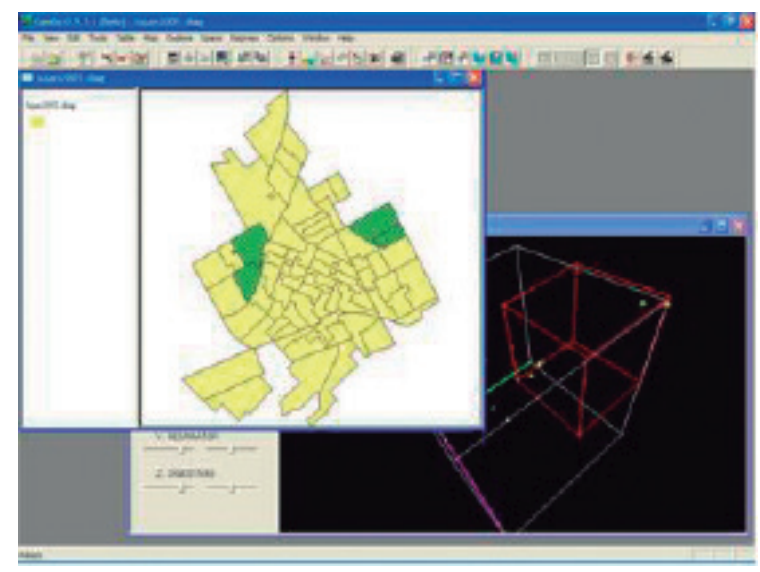

Aplicación del Análisis de Autocorrelación Espacial en el estudio de la distribución espacial trivariada de enfermedades

.Figura 4. Luján. Autocorrelación espacial de enfermedades infecciosas

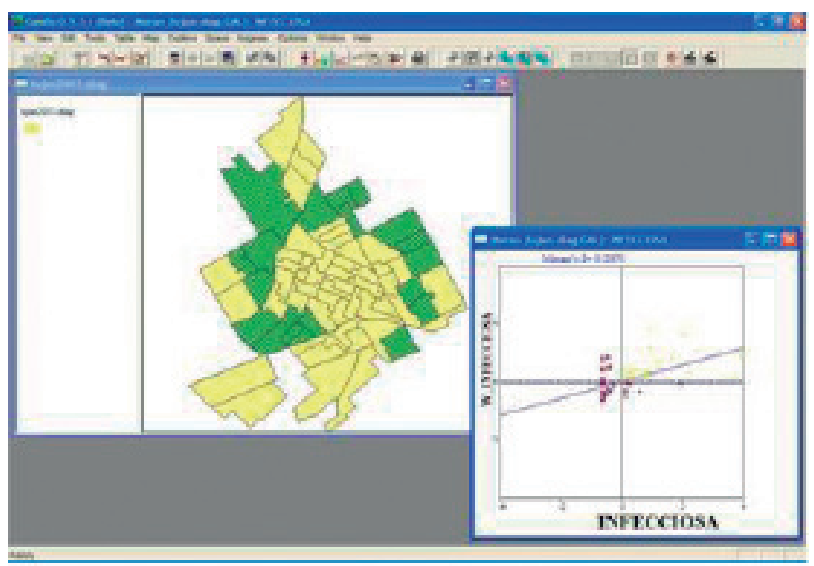


Figura 5. Test de aleatoriedad en la autocorrelación espacial

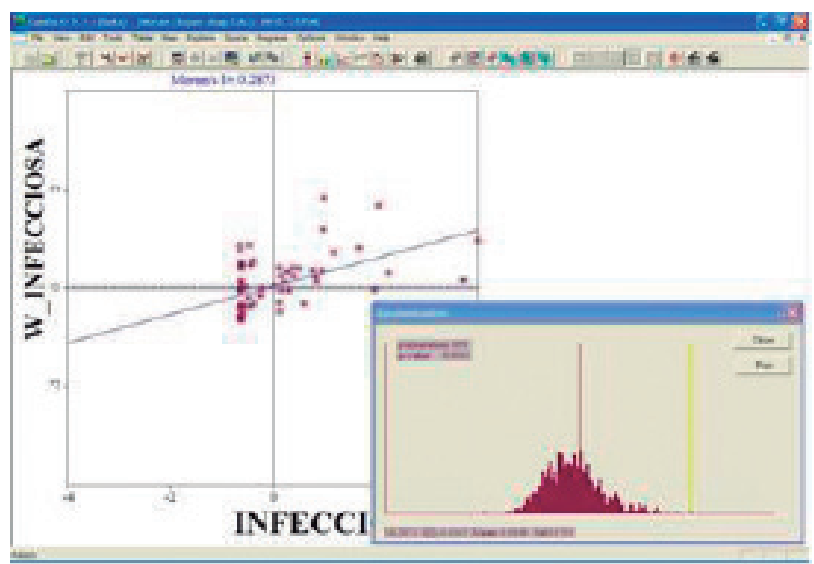

Figura 6. Luján. Cálculo de LISA y áreas de cúmulos

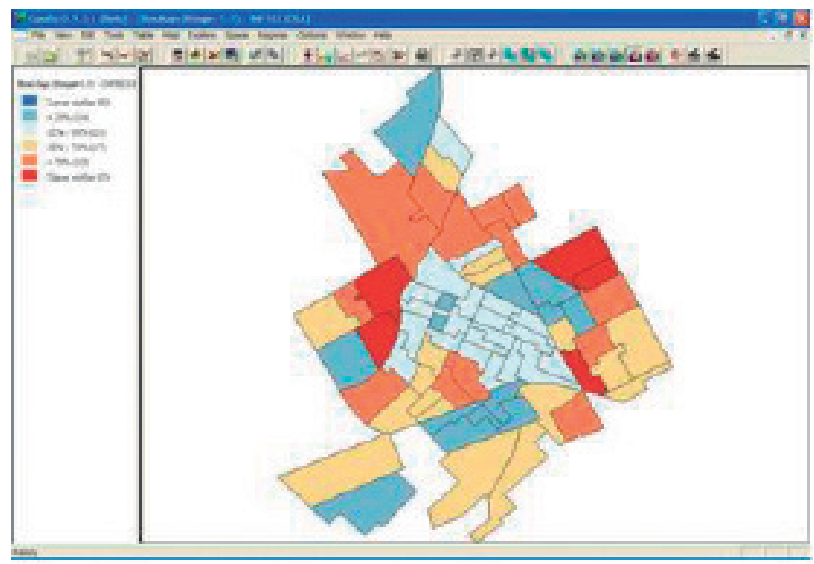

Aplicación del análisis multivariado en la correlación de diagnósticos para la determinación de agrupamientos a partir de los comportamientos espaciales comunes. 
Figura 7. Dendrograma del agrupamiento de variables

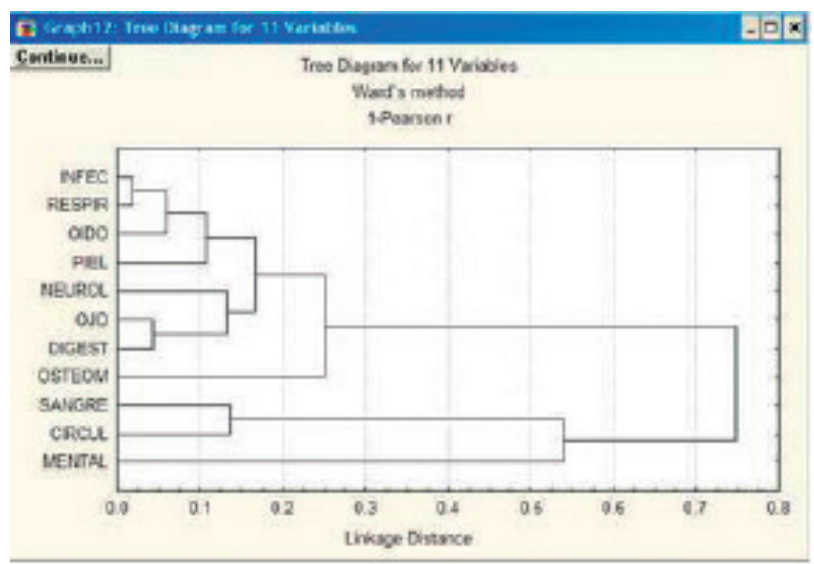

\section{APLICACIONES EN GEOGRAFÍA DE LOS SERVICIOS DE SALUD}

La aplicación de SIG+SADE en estudios de Geografía de los servicios de salud están orientados hacia aplicaciones de gran especificidad. Se incluyen las aplicaciones en evaluación multicriterio en la búsqueda de sitios candidatos para localizar nuevas instalaciones y el uso de modelos de localización-asignación que permiten evaluar la eficiencia y equidad espacial que produce cada una de ellas en el sistema espacial. Asimismo se encuentran aquí los análisis basados en la red, como los de áreas de influencia y caminos óptimos.

Los procedimientos aplicados en los proyectos de GESIG (Buzai, 2007a, 2007b, 2009, 2011, 2012 y 2013; Cruz, 2013) son los siguientes:

Aplicación de las técnicas de evaluación multicriterio para la determinación de sitios candidatos para la localización de nuevos centros de atención de salud. 
Figura 8. Luján. Resultado de riesgo mínimo

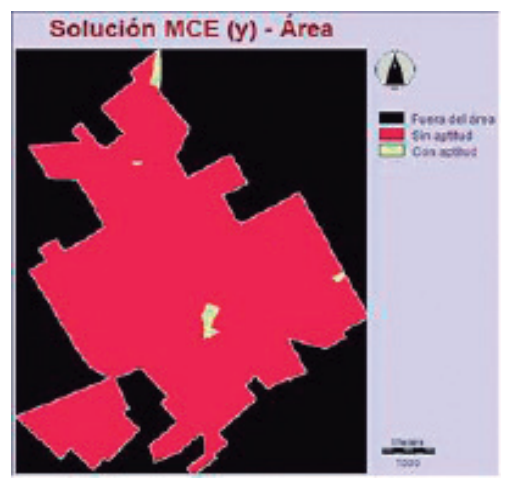

Figura 9. Luján. Resultado de riesgo creciente

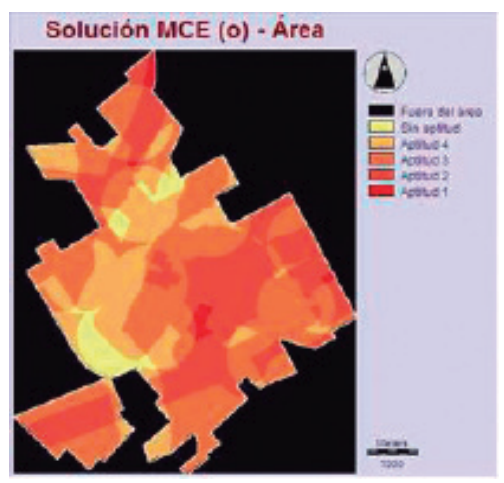

Resultados por combinación lineal ponderada con mayor peso hacia la población con Necesidades Básicas Insatisfechas (NBI) 
Figura 10. Ponderación por ranking recíproco: CAPS (3), Colectivos (2), Hidrografia (4), Población con NBI (1). Orientación hacia las zonas de pobreza.

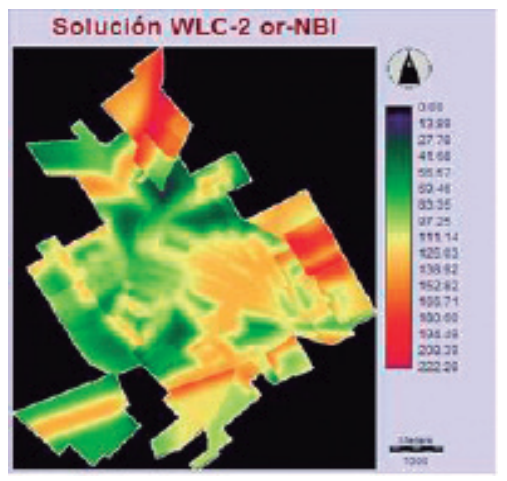

FIGURA 11. Resultado 10 con restricción. Representación con valores superiores a 150 puntos

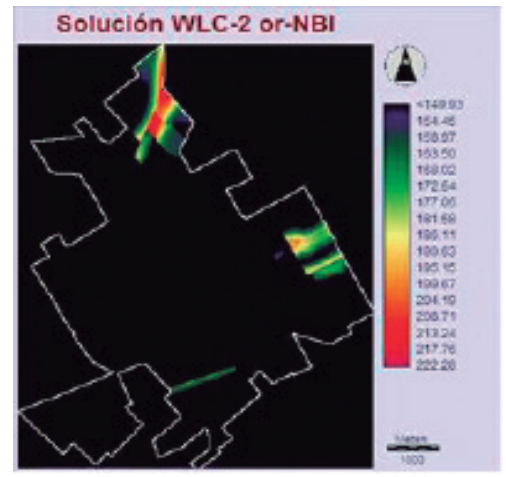

Resultados por localización multi-objetivo a partir del cual se obtiene un mapa con posibilidades diferenciadas. 
Figura 12. Resultado que combina orientaciones al NBI y a las vías de comunicación

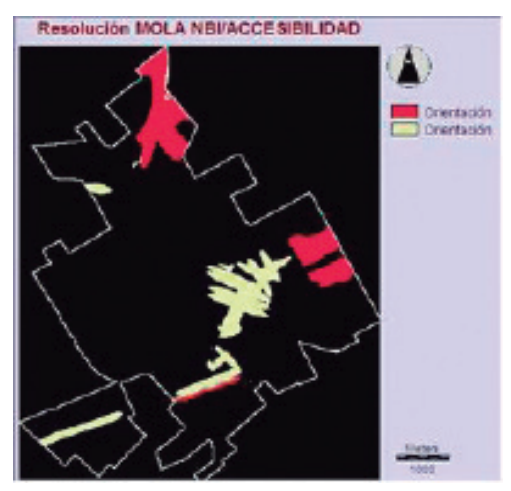

Aplicación de análisis de distancia para para la determinación de áreas de influencia de centros de atención de salud a partir de la distribución espacial real.

Figura 13. Cálculo de áreas de influencia sin restricción

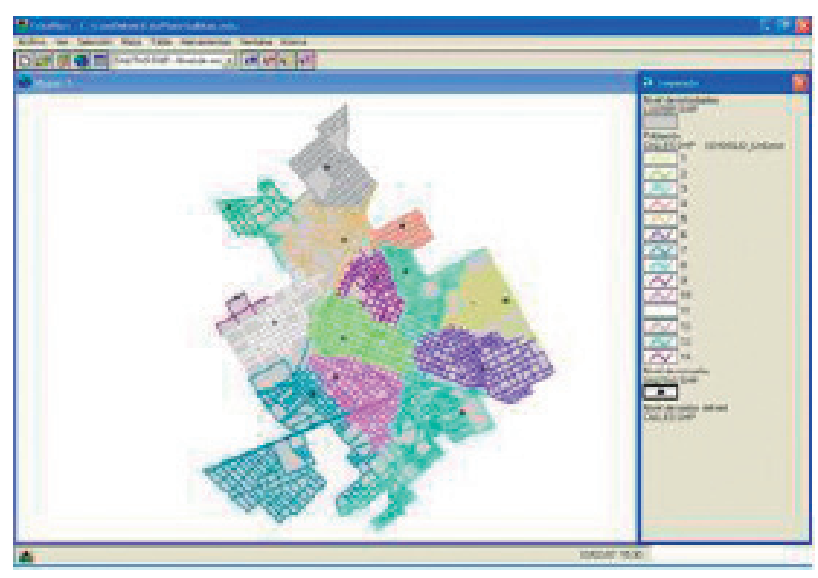


Figura 14. Cálculo de áreas de influencia con restricción de 1000 metros

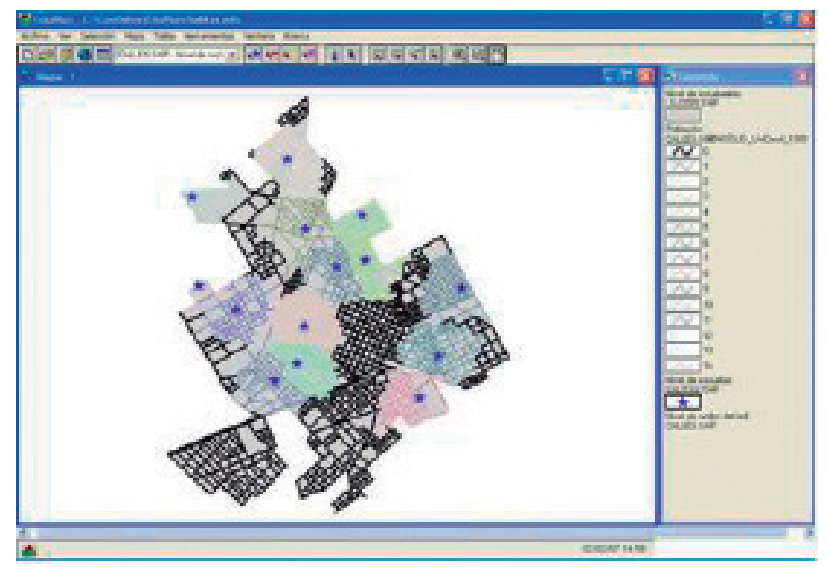

Aplicación de análisis de accesibilidad de la población hacia los centros de atención de salud a partir de la distribución espacial real.

Figura 15. Accesibilidad de la población total por distancia Manhattan

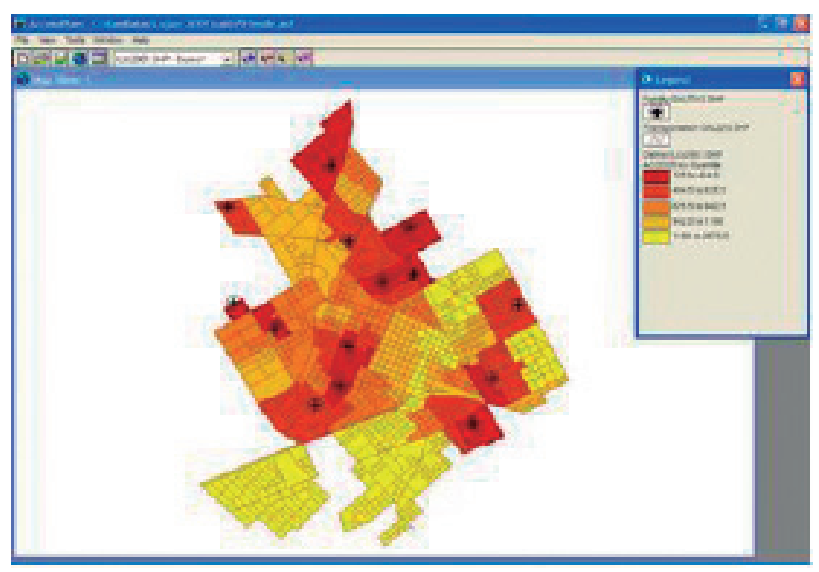

Aplicación del análisis de accesibilidad a partir del cálculo de densidades kernel. 
Figura 16. Distribución de la población total - método Kernel $\tau=1000$

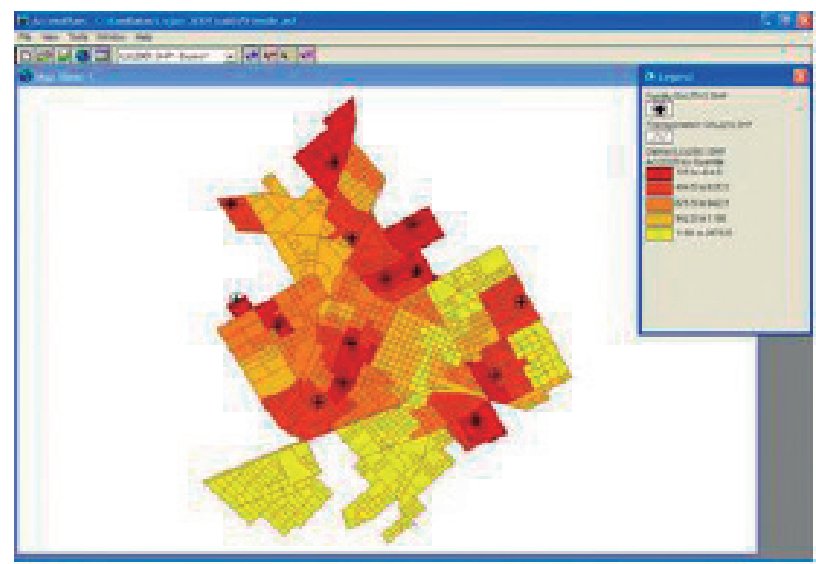

Figura 17. Distribución de la población con NBI - método Kernel $\tau=1000$

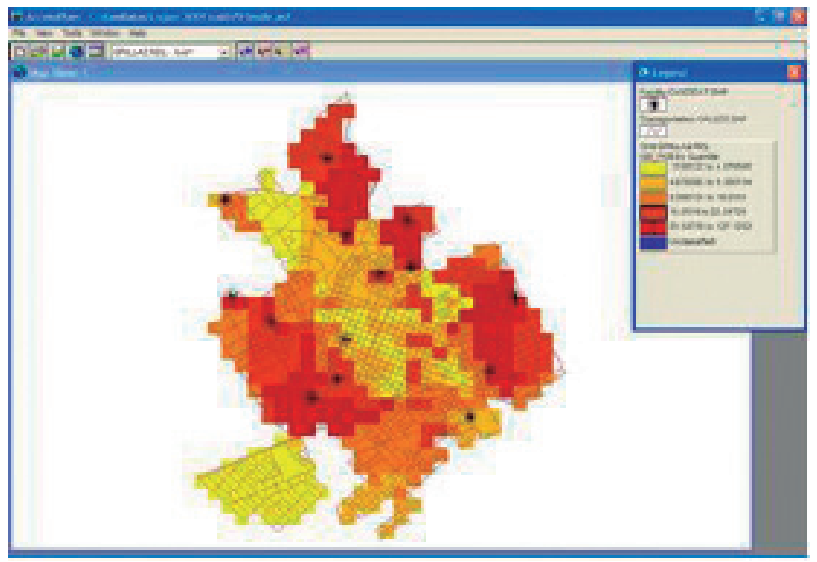

Aplicación de modelos de localización-asignación para la determinación de nuevos centros de atención de salud. 
Figura 18. Luján. Áreas de influencia de los CAPS

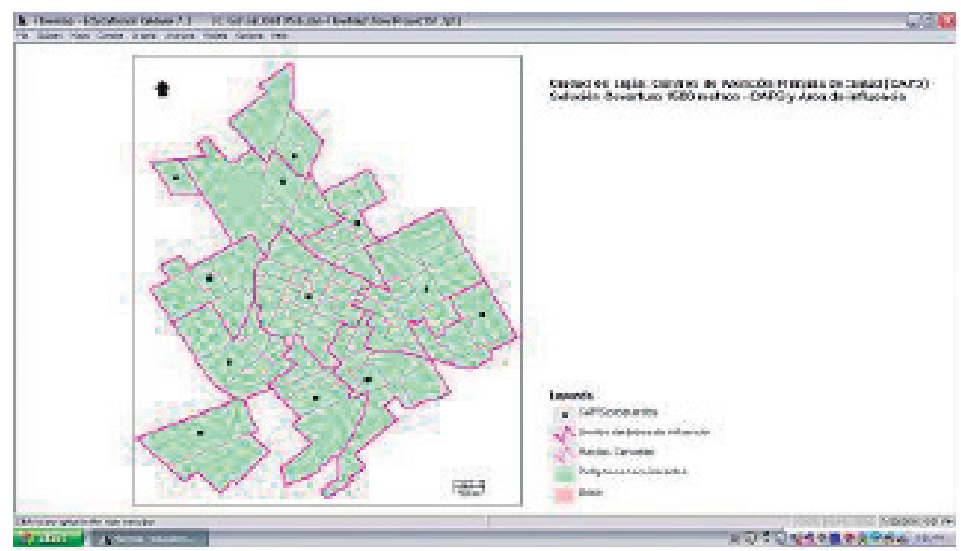

Figura 19. Luján. Asignación de demanda potencial a los CAPS

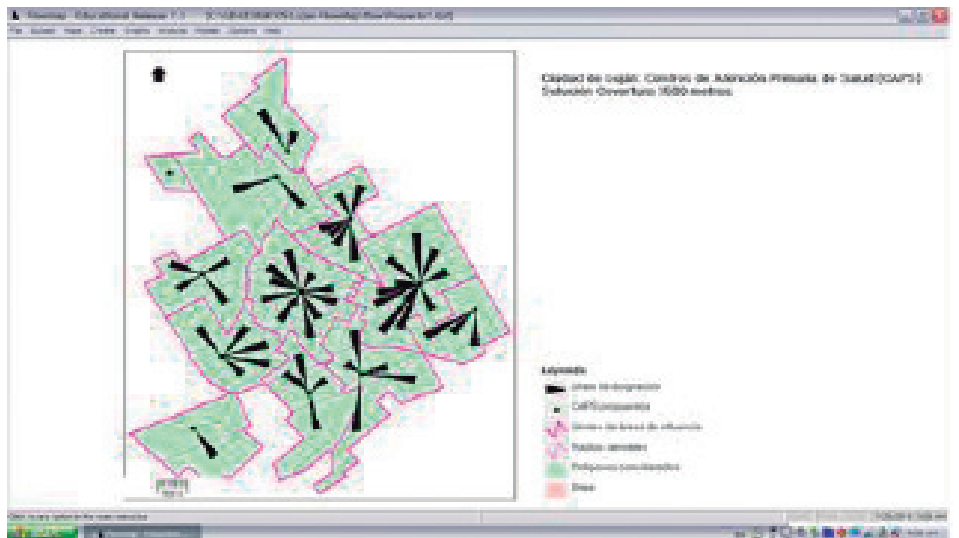

\section{CONSIDERACIONES FINALES}

La Geografía de la Salud es actualmente uno de los campos de estudio de mayor dinamismo al incorporar con claridad las tecnologías geoinformáticas y con ello obtener importantes resultados de aplicación. 
Su división interna en Geografía Médica y Geografía de los Servicios de salud le ha permitido clasificar eficientemente sus diferentes metodologías y lograr una muy correcta especificidad en cada aplicación. En el presente trabajo se ha presentado una variedad de posibilidades.

En la primera línea están los estudios correspondientes a la distribución de enfermedades a través del análisis univariado realizado a través de cartografía temática, el análisis bivariado a través del ESDA y el análisis de autocorrelación espacial. En la segunda, para el estudio de la localización de centros de atención se aplican técnicas de evaluación multicriterio en la búsqueda de sitios candidatos y modelos de localización-asignación en la determinación de sitios.

Conociendo la imposibilidad de agotar el tema, los ejemplos presentados, realizados en investigaciones del GESIG han intentado presentar una gran variedad de aplicaciones realizadas en la temática.

La Geografía de la Salud, a través de las actuales tendencias en Geografía Aplicada, contribuye para que los conceptos geográficos centrales puedan hacerse operativos en la búsqueda de soluciones socioespaciales. Las tecnologías digitales basadas en la cuantificación se encuentran disponibles para apoyar su logro.

\section{BIBLIOGRAFIA}

- Buzai, G.D. 2007a. Geografía de la Salud en la ciudad de Luján. Cuadernos de Trabajo 24. Departamento de Ciencias Sociales. Universidad Nacional de Luján. Luján.

- Buzai, G.D. (ed.) 2007b. Métodos cuantiativos en Geografía de la Salud. Programa de Estudios Geográficos. Universidad Nacional de Luján.

- Buzai, G.D. 2009. Sistemas de Información Geográfica en Geografía de la Salud. En: J.A. Pickenhayn (ed.) Salud y enfermedad en Geografía. Lugar Editorial. Buenos Aires. pp. 111-134.

- Buzai, G.D. 2011. Modelos de localización-asignación aplicados a servicios públicos urbanos. Análisis espacial de Centros de Atención Primaria de Salud (CAPS) en la ciudad de Luján, Argentina. Cuadernos de Geografía / Revista 
Colombiana de Geografía. 20(8):111-123.

- Buzai, G.D. 2012. Identificacao de sítios para localizacao de novos centros de atencao primária de saúde: aplicacao para a cidade de Luján, Argentina. Hygeia. 8(15):201-202.

- Buzai, G.D. 2013. Sistemas de Información Geográfica aplicados al estudio de la salud. Lugar Editorial. Buenos Aires.

- Buzai, G.D.; Baxendale, C.A. 2011. Análisis Socioespacial con Sistemas de Información Geográfica. Tomo 1: Perspectiva científica / temáticas de base raster. Lugar Editorial. Buenos Aires.

- Buzai, G.D.; Baxendale, C.A. 2012. Análisis Socioespacial con Sistemas de Información Geográfica. Tomo 2: Ordenamiento territorial / temáticas de base vectorial. Lugar Editorial. Buenos Aires.

- Cruz, M.R. 2012. Geografía de la Salud en el medio urbano. Diagnóstico socioespacial y morbilidad asistida en la ciudad de Luján. Editorial Académica Española. Saarbrucken.

- Cruz, M.R. 2013. Análisis de la distribución espacial de los servicios de salud de la Dirección de Asistencia Social y Medicina Integral de la Universidad Nacional de Luján. Propuesta de alternativa locacional fundada en los conceptos

de eficiencia y equidad espacial. (Tesis de Maestría). Universidad Nacional de Lanús. Lanús.

- Gatrell, A. 2002. Geographies of Health. Blackwell. London.

- Howe, G.M. 1985. La Geografía Médica. En: E.H.Brown (comp.) Geografía, pasado y futuro. Fondo de Cultura Económica. México. pp. 392-405.

- Olivera, A. 1994. Geografía de la Salud. Síntesis. Madrid. 